\title{
ON SOME REGULAR FRACTIONAL STURM-LIOUVILLE PROBLEMS WITH GENERALIZED DIRICHLET CONDITIONS
}

\author{
FATIMA-ZAHRA BENSIDHOUM AND HACEN DIB
}

Communicated by Neville Ford

ABSTRACT. The present work deals with some spectral properties of the problem

$(\mathcal{P})\left\{\begin{array}{c}D_{b,-}^{\alpha}\left(p(x) D_{a,+}^{\alpha} y\right)(x)+\lambda q(x) y(x)=0, \quad a<x<b, \\ \lim _{x \rightarrow a}(x-a)^{1-\alpha} y(x)=0=y(b),\end{array}\right.$

where $p, q \in C([a, b]), p(x)>0, q(x)>0$, for all $x \in[a, b]$ and $1 / 2<\alpha<1$. $D_{b,-}^{\alpha}$ and $D_{a,+}^{\alpha}$ are the right- and left-sided Riemann-Liouville fractional derivatives of order $\alpha \in(0,1)$, respectively. $\lambda$ is a scalar parameter.

First, we prove, using the spectral theory of linear compact operators, that this problem has an infinite sequence of real eigenvalues and the corresponding eigenfunctions form a complete orthonormal system in the Hilbert space $L_{q}^{2}[a, b]$. Then, we investigate some asymptotic properties of the spectrum as $\alpha \underset{<}{\longrightarrow}$. We give, in particular, the asymptotic expansion of the first eigenvalue.

1. Introduction. Sturm-Liouville theory has an important role in mathematics, physics and engineering. During the last few years, fractional Sturm-Liouville problems have appeared in the literature. They were introduced by Klimek and Agrawal $[\mathbf{7}, \mathbf{8}]$. Both RiemannLiouville and Caputo derivatives were used. These authors proved that the eigenvalues are real and the eigenfunctions are orthogonal by using a Green-type formula as in the classical case.

2010 AMS Mathematics subject classification. Primary 26A33, 34A08, Secondary 34B24, 34B27, 34L05, 34L10, 34L15, 47G10.

Keywords and phrases. Right- and left-sided Riemann-Liouville fractional derivatives, fractional Sturm-Liouville problem, fractional Green's function, HilbertSchmidt operators, min-max principle.

The first author is the corresponding author.

Received by the editors on June 24, 2015, and in revised form on February 28, 2016. 
Another important research area concerns the inverse problem in diffusion equations with fractional derivatives, namely, the problem of recovery of the diffusion potential from spectral data and the initial condition. Recently, much progress has been made in this direction by Tuan [18] and Bas [3], where one can find a wide number of references on the subject and many interesting comments. In [16], more problems have been presented. Also, similar problems to our $(\mathcal{P})$ have been considered by Bas et al. [2, 4] with singular coefficients $p(\cdot)$ as in Bessel-type equations.

The purpose of this paper is to investigate some basic spectral properties of the fractional Sturm-Liouville problem $(\mathcal{P})$ with generalized Dirichlet conditions. By generalized Dirichlet conditions, we mean

$$
\lim _{\substack{x \rightarrow a \\>}}(x-a)^{1-\alpha} y(x)=0=y(b) .
$$

Note that this kind of condition involves continuous as well as noncontinuous functions at the point $a$. For example,

$$
\lim _{\substack{x \rightarrow a \\>}} \ln (x-a)=-\infty \quad \text { but } \quad \lim _{\substack{x \rightarrow a \\>}}(x-a)^{1-\alpha} \ln (x-a)=0 .
$$

Obviously, Dirichlet conditions are a particular case of the generalized ones, and the limit in equation (1.1) implies that $y$ is in $L^{1}$.

We start by considering the problem $(\mathcal{P})$ on the domain $D o m$ where $y \in D o m$ if and only if $y$ verifies the following conditions:

$$
\begin{aligned}
& \text { (C1) } y \in C(] a, b]) \text { and } \lim _{\substack{x \rightarrow a \\
>}}(x-a)^{1-\alpha} y(x)=0 \\
& \text { (C2) } D_{a,+}^{\alpha} y \in C\left(\left[a, b[) \text { and } \lim _{\substack{x \rightarrow b \\
<}}(b-x)^{1-\alpha} D_{a,+}^{\alpha} y(x) \quad\right.\right. \text { exists, }
\end{aligned}
$$

with the aim of transform $(\mathcal{P})$ into an integral equation problem, which can be analyzed by classical tools of operator theory. Condition (C1) is nothing but the generalized Dirichlet condition at $x=a$. The second, (C2), will appear naturally further on in the calculation of the integral operator.

The number $\lambda$ is said to be an eigenvalue for problem $(\mathcal{P})$ if there exists a function in Dom, not identically zero, which satisfies the fractional regular Sturm-Liouville equation and the generalized Dirichlet conditions. 
In what follows, we prove that the set of eigenvalues of $(\mathcal{P})$ is real and infinite. This result partially answers a question in [16, page 9 , Remark 1], where the situation is quite similar. However, the simplicity of the eigenvalues is still an open problem.

The corresponding eigenfunctions form a complete orthonormal system in the Hilbert space $L_{q}^{2}[a, b]$ endowed with the usual scalar product and the associated norm:

$$
\langle u, v\rangle_{q}=\int_{a}^{b} u(x) \overline{v(x)} q(x) d x, \quad\|u\|_{q}=\sqrt{\langle u, u\rangle_{q}} .
$$

This result follows from the theory of linear compact operators and the fractional Green's function properties, following the approach in [10] adapted to our generalized boundary conditions. The second result investigates the asymptotic behavior of the first eigenvalue of $(\mathcal{P})$ when $\alpha \underset{<}{\rightarrow}$. This can be the starting point to put the classical and the fractional cases in the same theory.

2. Basic concepts. In this section, we recall some definitions and properties of Riemann-Liouville fractional integrals and fractional derivatives used below. For more details see $[\mathbf{1 1}, \mathbf{1 4}, \mathbf{1 5}, \mathbf{1 7}]$.

Definition 2.1 ([11, 15]). Let $f \in C([a, b])$. The right- and leftsided Riemann-Liouville fractional integrals of order $\alpha>0$ are defined, respectively, by

$$
\left(I_{b,-}^{\alpha} f\right)(x)=\frac{1}{\Gamma(\alpha)} \int_{x}^{b}(t-x)^{\alpha-1} f(t) d t
$$

and

$$
\left(I_{a,+}^{\alpha} f\right)(x)=\frac{1}{\Gamma(\alpha)} \int_{a}^{x}(x-t)^{\alpha-1} f(t) d t
$$

where $\Gamma$ is the Euler gamma function. 
Definition 2.2 ([11, 15]). The right- and left-sided Riemann-Liouville fractional derivatives of order $\alpha \in(0,1)$ are defined by:

$$
\left(D_{b,-}^{\alpha} f\right)(x)=-\frac{d}{d x}\left(I_{b,-}^{1-\alpha} f\right)(x)
$$

and

$$
\left(D_{a,+}^{\alpha} f\right)(x)=\frac{d}{d x}\left(I_{a,+}^{1-\alpha} f\right)(x)
$$

respectively.

Proposition 2.3 $([\mathbf{1 1}, \mathbf{1 5}, \mathbf{1 7}])$. Let $0<\alpha<1$ and $f \in C((a, b))$.

(i) $\left[\left(D_{b,-}^{\alpha} o I_{b,-}^{\alpha}\right) f\right](x)=f(x)$.

(ii) If $I_{b,-}^{1-\alpha} f \in A C([a, b])$, then we have

$$
\left[\left(I_{b,-}^{\alpha} o D_{b,-}^{\alpha}\right) f\right](x)=f(x)-\frac{(b-x)^{\alpha-1}}{\Gamma(\alpha)} \lim _{\substack{<\rightarrow b \\<}}\left(I_{b,-}^{1-\alpha} f\right)(x) .
$$

(iii) $\left[\left(D_{a,+}^{\alpha} o I_{a,+}^{\alpha}\right) f\right](x)=f(x)$.

(iv) If $I_{a,+}^{1-\alpha} f \in A C([a, b])$, then we have

$$
\left[\left(I_{a,+}^{\alpha} o D_{a,+}^{\alpha}\right) f\right](x)=f(x)-\frac{(x-a)^{\alpha-1}}{\Gamma(\alpha)} \lim _{x \rightarrow a}\left(I_{a,+}^{1-\alpha} f\right)(x) .
$$

Lemma 2.4 ([11, page 151, Lemma 3.2]). Let $0<\alpha<1$ and $y(x)$ be a Lebesgue measurable function on $[a, b]$.

(i) If there exists a limit

$$
\lim _{\substack{x \rightarrow a \\>}}(x-a)^{1-\alpha} y(x)=c, \quad c \in \mathbb{C},
$$

then the following limit also exists

$$
\left(I_{a,+}^{1-\alpha} y\right)(a+):=\lim _{\substack{x \rightarrow a \\>}}\left(I_{a,+}^{1-\alpha} y\right)(x)=c \Gamma(\alpha) .
$$

(ii) Symmetrically, if there exists a limit

$$
\lim _{\substack{x \rightarrow b \\<}}(b-x)^{1-\alpha} y(x)=d, \quad d \in \mathbb{C},
$$


then the following limit also exists

$$
\left(I_{b,-}^{1-\alpha} y\right)(b-):=\lim _{\substack{x \rightarrow b \\<}}\left(I_{b,-}^{1-\alpha} y\right)(x)=d \Gamma(\alpha) .
$$

Finally, we recall the following fractional integration by parts formula, proved in [17, page 46, Corollary 2] (with simple sufficient conditions).

Proposition 2.5. Let $f, g,\left(D_{a,+}^{\alpha} f\right),\left(D_{b,-}^{\alpha} g\right) \in C([a, b])$. Then

$$
\int_{a}^{b}\left(D_{a,+}^{\alpha} f\right)(x) g(x) d x=\int_{a}^{b} f(x)\left(D_{b,-}^{\alpha} g\right)(x) d x .
$$

\section{Main results.}

3.1. Spectral properties. In this subsection, we prove that problem $(\mathcal{P})$ admits an infinite sequence of eigenvalues, which are real and negative. Also, the normalized eigenfunctions form an orthonormal basis in $L_{q}^{2}[a, b]$.

Proposition 3.1. Let $\alpha \in((1 / 2), 1]$. Then

(i) for all $y \in$ Dom, problem $(\mathcal{P})$ is equivalent to

$$
y(x)=\lambda\left(T_{\alpha} y\right)(x),
$$

where $T_{\alpha}$ is the linear operator defined by

$$
\begin{aligned}
& T_{\alpha}: \text { Dom } \longrightarrow \text { Dom } \\
& \qquad y(\cdot) \longmapsto\left(T_{\alpha} y\right)(x)=\int_{a}^{b} G_{\alpha}(x, \tau) q(\tau) y(\tau) d \tau
\end{aligned}
$$

$G_{\alpha}(x, \tau)$ is the fractional Green's function defined by

$$
G_{\alpha}(x, \tau)= \begin{cases}\frac{\phi(b, x) \phi(b, \tau)}{\phi(b, b) \Gamma(\alpha)}-\frac{\phi(x, \tau)}{\Gamma(\alpha)}, & \text { if } a \leq \tau \leq x \\ \frac{\phi(b, x) \phi(b, \tau)}{\phi(b, b) \Gamma(\alpha)}-\frac{\phi(\tau, x)}{\Gamma(\alpha)}, & \text { if } x \leq \tau \leq b,\end{cases}
$$


and

$$
\begin{aligned}
\phi(u, v) & =I_{a,+}^{\alpha}\left(\frac{(u-v)^{\alpha-1}}{p(v)}\right)(v) \\
& =\frac{1}{\Gamma(\alpha)} \int_{a}^{v}(u-\tau)^{\alpha-1}(v-\tau)^{\alpha-1} \frac{d \tau}{p(\tau)} .
\end{aligned}
$$

(ii) $T_{\alpha}$ extends to a compact, self-adjoint operator on $L_{q}^{2}[a, b]$ and

$$
\operatorname{Ker}\left(T_{\alpha}\right)=\{0\} .
$$

(iii) All the eigenvalues of problem $(\mathcal{P})$ are negative.

Proof.

(i) By applying $I_{b,-}^{\alpha}$ to the equation

$$
D_{b,-}^{\alpha}\left(p(x) D_{a,+}^{\alpha} y\right)(x)+\lambda q(x) y(x)=0,
$$

we obtain

$$
\begin{aligned}
\left(D_{a,+}^{\alpha} y\right)(x)= & \frac{(b-x)^{\alpha-1}}{\Gamma(\alpha) p(x)} \lim _{\substack{x \rightarrow b \\
<}}\left(I_{b,-}^{1-\alpha} p(x) D_{a,+}^{\alpha} y\right)(x) \\
& -\frac{\lambda}{p(x)}\left(I_{b,-}^{\alpha} q(x) y(x)\right)(x) .
\end{aligned}
$$

Now, we apply the operator $I_{a,+}^{\alpha}$. Then

$$
\begin{aligned}
y(x)= & \frac{(x-a)^{\alpha-1}}{\Gamma(\alpha)} \lim _{x \rightarrow a}\left(I_{a,+}^{1-\alpha} y\right)(x)+\frac{C \phi(b, x)}{\Gamma(\alpha)} \\
& -\lambda I_{a,+}^{\alpha}\left(\frac{1}{p(x)}\left(I_{b,-}^{\alpha} q(x) y(x)\right)\right)(x),
\end{aligned}
$$

where, according to Proposition 2.3 (ii), we have

$$
C=\lim _{\substack{x \rightarrow b \\<}}\left(I_{b,-}^{1-\alpha} p(x) D_{a,+}^{\alpha} y\right)(x)
$$

and $\phi(b, x)$ is defined in equation (3.2).

- Note that the generalized Dirichlet condition

$$
\lim _{\substack{x \rightarrow a \\>}}(x-a)^{1-\alpha} y(x)=0
$$


implies that

$$
\lim _{\substack{x \rightarrow a \\>}}\left(I_{a,+}^{1-\alpha} y\right)(x)=0 .
$$

according to Lemma 2.4.

- The application of the second boundary value condition $y(b)=$ 0 gives

$$
C=\frac{\lambda \Gamma(\alpha)}{\phi(b, b)} \lim _{\substack{x \rightarrow b \\<b}} I_{a,+}^{\alpha}\left(\frac{1}{p(x)}\left(I_{b,-}^{\alpha} q(x) y(x)\right)\right)(x),
$$

where $\phi(b, b)=\lim _{x \rightarrow b} \phi(b, x)$ exists for $1 / 2<\alpha \leq 1$, since the integral

$$
\int_{a}^{b}(b-x)^{2 \alpha-2} \frac{d x}{p(x)}
$$

converges if and only if $2 \alpha-1>0$.

Let us compute the constant $C$. By using Fubini's theorem, we obtain:

$$
\begin{aligned}
I_{a,+}^{\alpha}\left(\frac{1}{p(x)}\left(I_{b,-}^{\alpha} q(x) y(x)\right)\right)(x)= & \frac{1}{\Gamma(\alpha)} \int_{a}^{x} \phi(x, \tau) q(\tau) y(\tau) d \tau \\
& +\frac{1}{\Gamma(\alpha)} \int_{x}^{b} \phi(\tau, x) q(\tau) y(\tau) d \tau
\end{aligned}
$$

Then we have

$$
\begin{aligned}
C & =\frac{\lambda \Gamma(\alpha)}{\phi(b, b)} \lim _{\substack{x \rightarrow b \\
<}} I_{a,+}^{\alpha}\left(\frac{1}{p(x)}\left(I_{b,-}^{\alpha} q(x) y(x)\right)\right)(x) \\
& =\frac{\lambda}{\phi(b, b)} \int_{a}^{b} \phi(b, \tau) q(\tau) y(\tau) d \tau .
\end{aligned}
$$

Thus, by substituting the first boundary value condition, the constant $C$ and the integral (3.5) into equation (3.3) we obtain the first implication.

We can easily verify that, for all $y \in$ Dom,

$$
T_{\alpha} y \in \text { Dom. }
$$


Then, the equivalence stated above follows immediately.

(ii) We can obtain the results from the properties of the fractional Green's function. The operator $T_{\alpha}$ defined on Dom can be continuously extended to $L_{q}^{2}[a, b]$ because Dom is dense, since it contains the space of infinitely differentiable functions with compact support. The compactness of $T_{\alpha}$ follows from the properties below. In fact,

(a) for all $(x, \tau) \in[a, b] \times[a, b], G_{\alpha}(x, \tau)$ is a continuous function because $\phi(x, \tau)$ is a continuous function in the domain $a \leq$ $\tau \leq x \leq b$ and $\lim _{\tau \rightarrow x} G_{\alpha}(x, \tau)=\lim _{\tau \rightarrow x} G_{\alpha}(x, \tau)$, the kernel $G_{\alpha} \in L_{q \otimes q}^{2}([a, b] \times[a, b])$ therefore $T_{\alpha}$ is a Hilbert-Schmidt operator, and thus compact (see $[\mathbf{5}, \mathbf{1 9}])$.

(b) For all $(x, \tau) \in[a, b] \times[a, b], G_{\alpha}(x, \tau)=G_{\alpha}(\tau, x)$, then $G$ is a symmetric function on $[a, b] \times[a, b]$ so the operator $T_{\alpha}$ is self-adjoint in $L_{q}^{2}[a, b]$.

(c) To prove that the kernel of $T_{\alpha}$ is reduced to $\{0\}$, it suffices to solve the equation

$$
\left(T_{\alpha} y\right)(x)=0, \quad \text { for all } x \in[a, b] .
$$

By application of the left- and right-sided Riemann-Liouville fractional derivatives, respectively, we obtain the result after some simple calculations.

(iii) Let us consider the fractional Sturm-Liouville equation

$$
D_{b,-}^{\alpha}\left(p(x) D_{a,+}^{\alpha} y\right)(x)+\lambda q(x) y(x)=0 .
$$

By multiplying this equation by $y(x)$ and integrating it on $[a, b]$ we get

$$
\int_{a}^{b} D_{b,-}^{\alpha}\left(p(x) D_{a,+}^{\alpha} y\right)(x) y(x) d x+\lambda \int_{a}^{b} q(x) y^{2}(x) d x=0
$$

Now, by applying Proposition 2.5 (2.5), which is also satisfied with the generalized Dirichlet conditions (1.1), we obtain

$$
\int_{a}^{b} p(x)\left(D_{a,+}^{\alpha} y\right)^{2}(x) d x+\lambda \int_{a}^{b} q(x) y^{2}(x) d x=0 ;
$$

hence, $\lambda$ must be negative. 
Theorem 3.2. Let us consider the fractional regular Sturm-Liouville problem $(\mathcal{P})$ for $1 / 2<\alpha<1$. Then

(i) The problem $(\mathcal{P})$ has an infinite countable set $\left\{\lambda_{n}\right\}_{n \geq 1}$ of real negative eigenvalues such that $\lim _{n \rightarrow \infty} \lambda_{n}=-\infty$, which can be ordered as:

$$
\cdots \leq \lambda_{n} \leq \cdots \leq \lambda_{3} \leq \lambda_{2} \leq \lambda_{1} \leq 0
$$

(ii) The normalized eigenfunctions $\left\{\phi_{n}\right\}_{n}$ corresponding to the eigenvalues $\left\{\lambda_{n}\right\}_{n}$ are orthogonal in $L_{q}^{2}[a, b]$, that is, for all $i \neq j$,

$$
\int_{a}^{b} \phi_{i}(x) \phi_{j}(x) q(x) d x=0 .
$$

(iii) Let $\left\{\phi_{n, j}\right\}_{j=1}^{d_{n}}$ be an orthonormal basis of $E_{n}$, the eigenspace corresponding to $\lambda_{n}\left(d_{n}=\operatorname{dim} E_{n}\right)$. The eigenfunctions $\left\{\phi_{n, j}\right\}_{n, j}$ form an orthonormal basis of the Hilbert space $L_{q}^{2}[a, b]$, that is, for all $y \in L_{q}^{2}[a, b]$,

$$
y=\lim _{N \rightarrow \infty} \sum_{n=1}^{N} \sum_{j=1}^{d_{n}}<y, \phi_{n, j}>_{q} \phi_{n, j} .
$$

Proof. Let $\lambda$ be a non-zero eigenvalue of $(\mathcal{P})$ and $\mu$ a non-zero eigenvalue of $T_{\alpha}$. Then, according to Proposition 3.1, we have

$$
(\mathcal{P}) \Longleftrightarrow \frac{1}{\lambda} y=T_{\alpha} y
$$

and

$$
\mu y=T_{\alpha} y \Longleftrightarrow\left\{\begin{array}{l}
D_{b,-}^{\alpha}\left(p(x) D_{a,+}^{\alpha} y\right)(x)+\frac{1}{\mu} q(x) y(x)=0 \\
\lim _{x \rightarrow a}(x-a)^{1-\alpha} y(x)=0=y(b) .
\end{array}\right.
$$

The homogeneous problem does not have a non-zero solution; hence, zero is neither an eigenvalue of $(\mathcal{P})$ nor an eigenvalue of $T_{\alpha}$.

Then, the non-zero eigenvalues of $(\mathcal{P})$ are exactly the inverses of those of $T_{\alpha}$. These last inverses are real countable, negative, tend to 
zero when $n \rightarrow \infty$ and they can be ordered as:

$$
\left|\mu_{1}\right| \geq\left|\mu_{2}\right| \geq\left|\mu_{3}\right| \geq \cdots \geq\left|\mu_{k}\right| \geq \cdots \rightarrow 0
$$

because $T_{\alpha}$ is self-adjoint and compact in $L_{q}^{2}[a, b]$. We can also compute the properties of the eigenfunctions.

Now, we prove the infinity of eigenvalues by contradiction. Let us assume that $T_{\alpha}$ has a finite sequence of non-zero eigenvalues. The spectral theorem of compact self-adjoint operators gives:

$$
L_{q}^{2}[a, b]=\left(\operatorname{Ker} T_{\alpha}\right) \oplus\left(\bigoplus_{k=1}^{N} E_{n}\right) .
$$

Since the corresponding eigenspaces $E_{1}, \ldots, E_{n}$ are finite-dimensional and, according to Proposition 3.1 we have $\operatorname{Ker} T_{\alpha}=\{0\}$, then $L_{q}^{2}[a, b]$ must be finite-dimensional, which is a contradiction.

Remark 3.3. The integral (3.4) converges if and only if $\alpha>1 / 2$. Thus, the operator $T_{\alpha}$ remains compact and self-adjoint even if $\alpha \geq 1$. But the corresponding fractional Sturm-Liouville problem must contain many more boundary conditions.

Recall the expression of the Hilbert-Schmidt norm of a compact integral operator on $L_{q}^{2}[a, b][\mathbf{1 9}]$. If

$$
(S y)(x)=\int_{a}^{b} K(x, t) y(t) q(t) d t,
$$

then

$$
\|S\|_{H S}^{2}=\int_{a}^{b} \int_{a}^{b}|K(x, t)|^{2} q(t) q(x) d t d x
$$

Proposition 3.4. All of the eigenvalues of problem $(\mathcal{P})$ verify:

$$
|\lambda| \geq \frac{1}{\left\|T_{\alpha}\right\|_{H S}} .
$$

Proof. If $\lambda$ is an eigenvalue, then there exists a $y \neq 0$ such that

$$
y=\lambda T_{\alpha} y
$$


SO

$$
\|y\|_{q}=|\lambda| \cdot\left\|T_{\alpha} y\right\|_{q} \leq|\lambda| \cdot\left\|T_{\alpha}\right\| \cdot\|y\|_{q} \leq|\lambda| \cdot\left\|T_{\alpha}\right\|_{H S} \cdot\|y\|_{q} \cdot
$$

Then

$$
|\lambda| \geq \frac{1}{\left\|T_{\alpha}\right\|_{H S}}
$$

Remark 3.5. The optimal disc free from eigenvalues has $1 /\left\|T_{\alpha}\right\|$ as the radius. But the operator norm is more difficult to compute than the Hilbert-Schmidt. In our case,

$$
\left\|T_{\alpha}\right\|_{H S}^{2}=\int_{a}^{b} \int_{a}^{b}\left|G_{\alpha}(x, t)\right|^{2} q(t) q(x) d t d x
$$

can be computed for explicit functions $p$ and $q$.

3.2. Asymptotic behavior. In this section, we prove that the first eigenvalue of $T_{\alpha}$ is close to the first eigenvalue of the following problem

$$
\left(\mathcal{P}_{1}\right)\left\{\begin{array}{l}
-\left(p(x) y^{\prime}\right)^{\prime}(x)+\lambda q(x) y(x)=0, \quad a<x<b \\
y(a)=0=y(b)
\end{array}\right.
$$

when $\alpha \underset{<}{\longrightarrow} 1$.

To prove this result, we need some lemmas and propositions.

Lemma 3.6. The $n$th derivative of $1 / \Gamma(\alpha)$ and $\Gamma(\alpha)$ have the following properties. For all $n \geq 0$ there exists $M_{n}>0$, for all $\alpha \in[1 / 2,1]$,

$$
\begin{gathered}
\left|\left(\frac{1}{\Gamma(\alpha)}\right)^{(n)}\right| \leq M_{n} \\
\text { and } \\
\left|(\Gamma(\alpha))^{(n)}\right| \leq M_{n} .
\end{gathered}
$$

Proof. The functions $1 / \Gamma(\cdot), \Gamma(\cdot) \in C^{\infty}(] 0,+\infty[)$, then they are bounded on compact sets, as well as all of their derivatives. 
Lemma 3.7. For $a \leq t \leq x \leq b$, we have the following property for all $\varepsilon>0$ and for all $0 \leq j \leq k$, there exists $C_{k, j}(\varepsilon)>0 / 1 / 2+\varepsilon<\alpha \leq 1$,

$$
\left|\int_{a}^{t}(x-\tau)^{\alpha-1} \ln ^{j}(x-\tau)(t-\tau)^{\alpha-1} \ln ^{k-j}(t-\tau) d \tau\right| \leq C_{k, j}(\varepsilon)
$$

Proof. Let us consider the function $f_{m}(\xi, \beta)=\xi^{\beta} \ln ^{m}(\xi)$ with $m \in \mathbb{N}$ and $0<\beta<\alpha-(1 / 2)$. It is not hard to see that $f_{m}(\xi, \beta)$ is uniformly bounded in $[0, c] \times[0, \alpha-1 / 2]$. Therefore,

$$
\begin{aligned}
& \left|\int_{a}^{t}(x-\tau)^{\alpha-1} \ln ^{j}(x-\tau)(t-\tau)^{\alpha-1} \ln ^{k-j}(t-\tau) d \tau\right| \\
& =\left|\int_{a}^{t}(x-\tau)^{\alpha-\beta-1} f_{j}((x-\tau), \beta)(t-\tau)^{\alpha-\beta-1} f_{k-j}((t-\tau), \beta) d \tau\right| \\
& \leq A_{k, j}(\varepsilon) \int_{a}^{t}(x-\tau)^{\alpha-\beta-1}(t-\tau)^{\alpha-\beta-1} d \tau \\
& \leq \frac{A_{k, j}(\varepsilon)}{(2 \alpha-2 \beta-1)} \sqrt{(x-a)^{2 \alpha-2 \beta-1}-(x-t)^{2 \alpha-2 \beta-1}} \cdot \sqrt{(t-a)^{2 \alpha-2 \beta-1}} \\
& \leq \frac{A_{k, j}(\varepsilon)}{(2 \alpha-2 \beta-1)}(b-a)^{2 \alpha-2 \beta-1}
\end{aligned}
$$

where we used the Cauchy-Schwartz inequality. By choosing $2 \beta=$ $\alpha-(1 / 2)$, we get

$$
\begin{aligned}
\left|\int_{a}^{t}(x-\tau)^{\alpha-1} \ln ^{j}(x-\tau)(t-\tau)^{\alpha-1} \ln ^{k-j}(t-\tau) d \tau\right| & \leq \frac{A_{k, j}(\varepsilon)}{\varepsilon} d_{\varepsilon}\left(\frac{1}{2}\right) \\
& =C_{k, j}(\varepsilon)
\end{aligned}
$$

where

$$
d_{\varepsilon}(\gamma)= \begin{cases}(b-a)^{\gamma} & \text { if } b \geq a+1 \\ (b-a)^{2 \gamma \varepsilon} & \text { if } b<a+1\end{cases}
$$

Lemma 3.8. Let $g$ be the function defined by

$$
g(\alpha)=\int_{a}^{b}(b-\tau)^{2 \alpha-2} \frac{d \tau}{p(\tau)}
$$

Then $g$ has the following properties: 
(i) for all $\varepsilon>0$, there exists

$$
K_{\min }, K_{\max }>0 /(1 / 2)+\varepsilon<\alpha \leq 1 \Longrightarrow K_{\min } \leq|g(\alpha)| \leq K_{\max } .
$$

(ii) For all $\varepsilon>0$ and $n \geq 0$, there exists

$$
\eta_{n}>0 /(1 / 2)+\varepsilon<\alpha \leq 1 \Longrightarrow\left|g^{(n)}(\alpha)\right| \leq \eta_{n} .
$$

(iii) For all $\varepsilon>0$ and $n \geq 0$, there exists

$$
\left.\delta_{n}>0 /(1 / 2)+\varepsilon<\alpha \leq 1 \Longrightarrow \mid 1 / g(\alpha)\right)^{(n)} \mid \leq \delta_{n} .
$$

Proof. Let

$$
g(\alpha)=\int_{a}^{b}(b-\tau)^{2 \alpha-2} \frac{d \tau}{p(\tau)} .
$$

Since $p \in C([a, b])$ and $p(t)>0$, then

$$
\frac{1}{M}=\frac{1}{\max _{\tau \in[a, b]} p(\tau)} \leq \frac{1}{p(\tau)} \leq \frac{1}{\min _{\tau \in[a, b]} p(\tau)}=\frac{1}{m}
$$

hence,

$$
\frac{(b-a)^{2 \alpha-1}}{M(2 \alpha-1)} \leq|g(\alpha)| \leq \frac{(b-a)^{2 \alpha-1}}{m(2 \alpha-1)} .
$$

Letting $\varepsilon>0 /(1 / 2)+\varepsilon<\alpha \leq 1$, we conclude that

$$
\frac{c_{\varepsilon}}{M} \leq|g(\alpha)| \leq \frac{d_{\varepsilon}(1)}{m(2 \varepsilon)}
$$

where

$$
c_{\varepsilon}= \begin{cases}(b-a)^{2 \varepsilon} & \text { if } b \geq a+1 \\ b-a & \text { if } b<a+1,\end{cases}
$$

which gives the first property.

For $g^{(n)}(\alpha)$, we have

$$
\begin{aligned}
g^{(n)}(\alpha) & =\int_{a}^{b} 2^{n} \ln ^{n}(b-\tau)(b-\tau)^{2 \alpha-2} \frac{d \tau}{p(\tau)} \\
& =\int_{a}^{b} 2^{n} f_{n}(b-\tau, \beta)(b-\tau)^{2 \alpha-\beta-2} \frac{d \tau}{p(\tau)},
\end{aligned}
$$


and then

$$
\left|g^{(n)}(\alpha)\right| \leq \frac{2^{n}}{m} A_{n}(\varepsilon) \frac{(b-a)^{2 \alpha-\beta-1}}{2 \alpha-\beta-1} .
$$

Now, we choose $\beta=\alpha-1 / 2$ to obtain

$$
\left|g^{(n)}(\alpha)\right| \leq \frac{2^{n}}{m \varepsilon} A_{n}(\varepsilon) d_{\varepsilon}\left(\frac{1}{2}\right)=\eta_{n}
$$

Finally, we can compute $(1 / g(\alpha))^{(n)}$ by application of the $n$th derivative of composite functions (see [13]) to the following functions:

$$
f(\alpha)=\frac{1}{\alpha} \quad \text { and } \quad g(\alpha)=\int_{a}^{b}(b-\tau)^{2 \alpha-2} \frac{d \tau}{p(\tau)} .
$$

Thus,

$$
(f \circ g)^{(n)}(\alpha)=\sum_{k=1}^{n} f^{(k)}(g(\alpha)) \sum_{l=0}^{k} \frac{(-1)^{k-l}}{l !(k-l) !} g^{k-l}(\alpha)\left(g^{l}(\alpha)\right)^{(n)}
$$

where for all $k / 1 \leq k \leq n$,

$$
f^{(k)}(g(\alpha))=\frac{(-1)^{k} k !}{g^{k+1}(\alpha)}
$$

and for all $l / 0 \leq l \leq n, j_{0}=0$ and $j_{l+1}=n$, we have

$$
\left(g^{l}(\alpha)\right)^{(n)}=\sum_{j_{0}=0}^{j_{1}} \sum_{j_{1}=0}^{j_{2}} \sum_{j_{2}=0}^{j_{3}} \cdots \sum_{j_{l}=0}^{j_{l+1}}\left[\prod_{i=1}^{l}\left(\left(\begin{array}{c}
j_{i+1} \\
j_{i}
\end{array}\right)(g(\alpha))^{\left(j_{i+1}-j_{i}\right)}\right)\right],
$$

which can easily be proved by induction.

By substituting (3.11) and (3.12) into (3.10) we obtain:

$$
\begin{aligned}
(f \circ g)^{(n)}(\alpha)=\sum_{k=1}^{n} \sum_{l=0}^{k}\left(\begin{array}{l}
k \\
l
\end{array}\right) & \frac{(-1)^{l}}{g^{l+1}(\alpha)} \sum_{j_{0}=0}^{j_{1}} \ldots \\
& \sum_{j_{l}=0}^{j_{l+1}}\left[\prod_{i=1}^{l}\left(\left(\begin{array}{c}
j_{i+1} \\
j_{i}
\end{array}\right)(g(\alpha))^{\left(j_{i+1}-j_{i}\right)}\right)\right],
\end{aligned}
$$

where for all $l / 0 \leq l \leq n, j_{l+1}=n$. 
Using triangular inequality, the first property of $g(\alpha)$ and property (3.9), we conclude that, for all $\varepsilon>0$ and $n \geq 0$, there exists:

$$
\delta_{n}>0, / \frac{1}{2}+\varepsilon<\alpha \leq 1 \Longrightarrow\left|(f \circ g)^{(n)}(\alpha)\right| \leq \delta_{n} .
$$

Proposition 3.9. For $a \leq t \leq x \leq b$ and

$$
\phi(x, t)=\frac{1}{\Gamma(\alpha)} \int_{a}^{t}(t-\tau)^{\alpha-1}(x-\tau)^{\alpha-1} \frac{d \tau}{p(\tau)},
$$

we have for all $\varepsilon>0$ and $n \geq 0$, there exists:

$$
K_{n}>0 / \frac{1}{2}+\varepsilon<\alpha \leq 1 \Longrightarrow\left|\frac{\partial^{n}}{\partial \alpha^{n}} \phi(x, t)\right| \leq K_{n} .
$$

For all $\varepsilon>0$ and $n \geq 0$, there exists

$$
B_{n}>0 / \frac{1}{2}+\varepsilon<\alpha \leq 1 \Longrightarrow\left|\frac{\partial^{n}}{\partial \alpha^{n}}\left(\frac{1}{\phi(b, b)}\right)\right| \leq B_{n} .
$$

Proof.

$$
\text { - } \frac{\partial^{n}}{\partial \alpha^{n}} \phi(x, t)=\frac{\partial^{n}}{\partial \alpha^{n}}\left(\frac{1}{\Gamma(\alpha)} \int_{a}^{t}(t-\tau)^{\alpha-1}(x-\tau)^{\alpha-1} \frac{d \tau}{p(\tau)}\right)
$$

by twice applying Leibniz's formula, we obtain

$$
\begin{aligned}
& =\sum_{k=0}^{n}\left(\begin{array}{l}
n \\
k
\end{array}\right)\left(\frac{1}{\Gamma(\alpha)}\right)^{(n-k)}\left(\int_{a}^{t}(t-\tau)^{\alpha-1}(x-\tau)^{\alpha-1} \frac{d \tau}{p(\tau)}\right)^{(k)} \\
& =\sum_{k=0}^{n} \sum_{j=0}^{k}\left(\begin{array}{l}
n \\
k
\end{array}\right)\left(\begin{array}{l}
k \\
j
\end{array}\right)\left(\frac{1}{\Gamma(\alpha)}\right)^{(n-k)} \\
& \left(\int_{a}^{t}(x-\tau)^{\alpha-1} \ln ^{j}(x-\tau)(t-\tau)^{\alpha-1} \ln ^{k-j}(t-\tau) \frac{d \tau}{p(\tau)}\right) .
\end{aligned}
$$

Then, according to the triangular inequality, we obtain

$$
\begin{aligned}
\left|\frac{\partial^{n}}{\partial \alpha^{n}} \phi(x, t)\right| & \leq \sum_{k=0}^{n} \sum_{j=0}^{k}\left(\begin{array}{l}
n \\
k
\end{array}\right)\left(\begin{array}{l}
k \\
j
\end{array}\right)\left|\left(\frac{1}{\Gamma(\alpha)}\right)^{(n-k)}\right| \\
& \left|\int_{a}^{t}(x-\tau)^{\alpha-1} \ln ^{j}(x-\tau)(t-\tau)^{\alpha-1} \ln ^{k-j}(t-\tau) \frac{d \tau}{p(\tau)}\right| .
\end{aligned}
$$


The facts that $p \in C([a, b])$ and $p(\tau)>0$ give

$$
\frac{1}{p(\tau)} \leq \frac{1}{\min _{\tau \in[a, b]} p(\tau)}=\frac{1}{m} \text {. }
$$

Then, by Lemmas 3.6 and 3.7, we obtain for all $\varepsilon>0$ and $n \geq 0$ that there exists

$$
\begin{gathered}
K_{n}=\frac{1}{m} \sum_{k=0}^{n} \sum_{j=0}^{k}\left[\left(\begin{array}{l}
n \\
k
\end{array}\right)\left(\begin{array}{l}
k \\
j
\end{array}\right) M_{n-k} C_{k, j}\right]>0 / \\
\frac{1}{2}+\varepsilon<\alpha \leq 1 \Longrightarrow\left|\frac{\partial^{n}}{\partial \alpha^{n}} \phi(x, t)\right| \leq K_{n} .
\end{gathered}
$$

- We have

$$
\phi(b, b)=\frac{1}{\Gamma(\alpha)} \int_{a}^{b}(b-\tau)^{2 \alpha-2} \frac{d \tau}{p(\tau)} \Longleftrightarrow \frac{1}{\phi(b, b)}=\frac{\Gamma(\alpha)}{g(\alpha)} .
$$

Then, by application of Leibniz's formula, we obtain

$$
\frac{\partial^{n}}{\partial \alpha^{n}}\left(\frac{1}{\phi(b, b)}\right)=\sum_{k=0}^{n}\left(\begin{array}{l}
n \\
k
\end{array}\right)(\Gamma(\alpha))^{(n-k)}\left(\frac{1}{g(\alpha)}\right)^{(k)} .
$$

Hence, from properties (3.7) and (3.13) we obtain the desired result.

Proposition 3.10. Consider once more the operator $T_{\alpha}$,

$$
\left(T_{\alpha} y\right)(x)=\int_{a}^{b} G_{\alpha}(x, \tau) q(\tau) y(\tau) d \tau
$$

where $G_{\alpha}$ is defined in equation (3.1). Then $T_{\alpha}^{(n)}=\partial^{n} /\left(\partial \alpha^{n}\right) T_{\alpha}$ is uniformly bounded for $(1 / 2)+\varepsilon \leq \alpha \leq 1$, that is, for all $\varepsilon>0$ and $n \geq 0$, there exists:

$$
\theta_{n}>0 / \frac{1}{2}+\varepsilon \leq \alpha \leq 1 \Longrightarrow\left\|T_{\alpha}^{(n)}\right\| \leq \theta_{n} .
$$

Proof. We have

$$
\left(T_{\alpha}^{(n)} y\right)(x)=\int_{a}^{b} G_{\alpha}^{(n)}(x, \tau) q(\tau) y(\tau) d \tau
$$


and

- for $a \leq \tau \leq x$, then

$$
\begin{aligned}
G_{\alpha}^{(n)}(x, \tau) & =\sum_{m=0}^{n}\left(\begin{array}{c}
n \\
m
\end{array}\right)\left(\frac{1}{\Gamma(\alpha)}\right)^{(n-m)} \\
\cdot\left[\sum_{l=0}^{m}\left(\left(\begin{array}{c}
m \\
l
\end{array}\right) \phi^{(m-l)}(b, x) \sum_{k=0}^{l}\left(\begin{array}{l}
l \\
k
\end{array}\right) \phi^{(l-k)}(b, \tau)\left(\frac{1}{\phi(b, b)}\right)^{(k)}\right)\right. & \left.-\phi^{(m)}(x, \tau)\right],
\end{aligned}
$$

and

- for $x \leq \tau \leq b$, then

$$
\begin{array}{r}
G_{\alpha}^{(n)}(x, \tau)=\sum_{m=0}^{n}\left(\begin{array}{c}
n \\
m
\end{array}\right)\left(\frac{1}{\Gamma(\alpha)}\right)^{(n-m)} \\
\cdot\left[\sum_{l=0}^{m}\left(\left(\begin{array}{c}
m \\
l
\end{array}\right) \phi^{(m-l)}(b, \tau) \sum_{k=0}^{l}\left(\begin{array}{l}
l \\
k
\end{array}\right) \phi^{(l-k)}(b, x)\left(\frac{1}{\phi(b, b)}\right)^{(k)}\right)\right. \\
\left.-\phi^{(m)}(\tau, x)\right],
\end{array}
$$

obtained by Leibniz's formula.

Then, by the Cauchy-Schwartz inequality, the fact that $q \in C([a, b])$ and $q(\tau)>0$, we can easily prove that

$$
\left\|T_{\alpha}^{(n)}\right\| \leq\left\|T_{\alpha}^{(n)}\right\|_{H . S .} \leq \sup _{\tau \in[a, b]} q(\tau) \sqrt{\int_{a}^{b} \int_{a}^{b}\left[G_{\alpha}^{(n)}(x, \tau)\right]^{2} d \tau d x .}
$$

Thus, by application of Lemma 3.6, Proposition 3.9 and formula (3.16) of $G_{\alpha}^{(n)}$, we obtain result (3.15).

Now, we can state the second main result.

\section{Theorem 3.11.}

(i) Let $m \geq 0$ be a fixed integer. Then, $T_{\alpha}$ is an $(m+1)$ th differentiable operator with respect to $\alpha$ at $\alpha=1$, and we have the 
following expansion

$$
T_{\alpha} y=\sum_{k=0}^{m} \frac{\left.\left(T_{\alpha}^{(k)} y\right)\right|_{\alpha=1}}{k !}(\alpha-1)^{k}+R_{m}(\alpha) y,
$$

where the Lagrange reminder is expressed by

$$
R_{m}(\alpha) y=\left.\frac{(\alpha-1)^{m+1}}{(m+1) !}\left(T_{\alpha}^{(m+1)} y\right)\right|_{\alpha=\xi}, \quad \alpha<\xi<1,
$$

and

$$
\left\|R_{m}(\alpha)\right\|=o\left(|\alpha-1|^{m}\right), \quad \alpha \underset{<}{\rightarrow} 1
$$

(ii) If $\mu_{1}^{-}(\alpha)$ is the first negative eigenvalue of the operator $T_{\alpha}$, then

$$
\lim _{\alpha \rightarrow 1} \mu_{1}^{-}(\alpha)=\mu_{1}^{-}(1),
$$

where $\mu_{1}^{-}(1)$ is the first negative eigenvalue of the operator $T_{1}$.

\section{Proof.}

(i) From expression (3.1) of the kernel $G_{\alpha}(x, \tau)$, one can see that $G_{\alpha}(x, \tau)$ is $C^{\infty}$ in any small neighborhood of $\alpha=1$. So we can write the Taylor formula with Lagrange reminder at this point as:

$$
\left(T_{\alpha} y\right)(x)=\sum_{k=0}^{m} \frac{\left.\left(T_{\alpha}^{(k)} y\right)(x)\right|_{\alpha=1}}{k !}(\alpha-1)^{k}+R_{m}(\alpha)
$$

where

$$
\left.\left(T_{\alpha}^{(k)} y\right)(x)\right|_{\alpha=1}=\left.\int_{a}^{b} G_{\alpha}^{(k)}(x, \tau)\right|_{\alpha=1} q(\tau) y(\tau) d \tau
$$

and $R_{m}(\alpha)$ is the Lagrange reminder defined in equation (3.18).

Then,

$$
\left\|R_{m}(\alpha)\right\| \leq\left\|T_{\xi}^{(m+1)}\right\| \cdot \frac{|\alpha-1|^{m+1}}{(m+1) !}, \quad \alpha<\xi<1 .
$$


By applying property (3.15), we obtain that there exists:

$$
\theta_{m+1}>0 /\left\|R_{m}(\alpha)\right\| \leq \theta_{m+1} \cdot \frac{|\alpha-1|^{m+1}}{(m+1) !},
$$

which gives

$$
\left\|R_{m}(\alpha)\right\|=o\left(|\alpha-1|^{m}\right), \quad \alpha \underset{<}{\rightarrow} 1
$$

(ii) Let us take the previous expansion of $T_{\alpha}$ equation (3.17) with $m=0$. Then

$$
\begin{aligned}
\left(T_{\alpha} y\right)(x)=\left(T_{1} y\right)(x)+(\alpha-1) \cdot\left(T_{\xi}^{\prime} y\right)(x) & \\
& \Longleftrightarrow\left|\left\langle T_{\alpha} y, y\right\rangle_{q}-\left\langle T_{1} y, y\right\rangle_{q}\right|=|\alpha-1| \cdot\left|\left\langle T_{\xi}^{\prime} y, y\right\rangle_{q}\right| .
\end{aligned}
$$

However,

$$
\left|\left\langle T_{\xi}^{\prime} y, y\right\rangle_{q}\right| \leq\left\|T_{\xi}^{\prime}\right\| \cdot\|y\|_{q} \leq\left\|T_{\xi}^{\prime}\right\|, \quad \text { for all } y /\|y\|_{q}=1 .
$$

Hence,

$$
\begin{aligned}
& \left|\left\langle T_{\alpha} y, y\right\rangle_{q}-\left\langle T_{1} y, y\right\rangle_{q}\right| \leq|\alpha-1| \cdot\left\|T_{\xi}^{\prime}\right\| \\
& \Longrightarrow-|\alpha-1| \cdot\left\|T_{\xi}^{\prime}\right\| \leq\left\langle T_{\alpha} y, y\right\rangle_{q}-\left\langle T_{1} y, y\right\rangle_{q} \leq|\alpha-1| \cdot\left\|T_{\xi}^{\prime}\right\| \\
& \Longrightarrow-|\alpha-1| \cdot\left\|T_{\xi}^{\prime}\right\|+\left\langle T_{1} y, y\right\rangle_{q} \leq\left\langle T_{\alpha} y, y\right\rangle_{q} \leq\left\langle T_{1} y, y\right\rangle_{q} \\
& \quad+|\alpha-1| \cdot\left\|T_{\xi}^{\prime}\right\| \\
& \quad \Longrightarrow-|\alpha-1| \cdot\left\|T_{\xi}^{\prime}\right\|+\inf _{\|y\|_{q}=1}\left\langle T_{1} y, y\right\rangle_{q} \leq \inf _{\|y\|_{q}=1}\left\langle T_{\alpha} y, y\right\rangle_{q}
\end{aligned}
$$

and

$$
\begin{aligned}
& \inf _{\|y\|_{q}=1}\left\langle T_{\alpha} y, y\right\rangle_{q} \leq \inf _{\|y\|_{q}=1}\left\langle T_{1} y, y\right\rangle_{q}+|\alpha-1| \cdot\left\|T_{\xi}^{\prime}\right\| \\
& \quad \Longrightarrow-|\alpha-1| \cdot\left\|T_{\xi}^{\prime}\right\|+\mu_{1}^{-}(1) \leq \mu_{1}^{-}(\alpha) \leq \mu_{1}^{-}(1)+|\alpha-1| \cdot\left\|T_{\xi}^{\prime}\right\| .
\end{aligned}
$$

From this and the fact that $T_{\xi}^{\prime}$ verifies $(3.15)$, we obtain

$$
\lim _{\substack{\alpha \rightarrow 1 \\<}} \mu_{1}^{-}(\alpha)=\mu_{1}^{-}(1),
$$

and consequently,

$$
\lim _{\substack{\alpha \rightarrow 1 \\<}} \lambda_{1}^{-}(\alpha)=\lambda_{1}^{-}(1) .
$$


Remark 3.12. We think that the previous expansion of $T_{\alpha}$ can be of great importance in the expression of the next terms in asymptotic development of $\mu_{1}^{-}(\alpha)$.

4. Illustrative example. Let us show a simple example with $p \equiv 1$, $q \equiv 1$ and $[a, b]=[0,1]$. Then, our regular fractional Sturm-Liouville problem reduces to

$$
\left\{\begin{array}{l}
\left(D_{1,-}^{\alpha} D_{0,+}^{\alpha}\right) y(x)+\lambda y(x)=0, \quad \frac{1}{2}<\alpha<1,0<x<1 \\
\lim _{\substack{x \rightarrow 0 \\
>}} x^{1-\alpha} y(x)=0=y(1),
\end{array}\right.
$$

and the "inverse" compact operator defined in $L^{2}([0,1])$ is

$$
\left(T_{\alpha} y\right)(x)=\int_{0}^{1} G_{\alpha}(x, \tau) y(\tau) d \tau,
$$

where the fractional Green's function is

$$
G_{\alpha}(x, \tau)= \begin{cases}(2 \alpha-1) \varphi(1, x) \varphi(1, \tau)-\varphi(x, \tau) & \text { if } 0 \leq \tau \leq x \\ (2 \alpha-1) \varphi(1, x) \varphi(1, \tau)-\varphi(\tau, x) & \text { if } x \leq \tau \leq 1\end{cases}
$$

with

$$
\begin{aligned}
& \varphi(\tau, x)=\frac{1}{\Gamma(\alpha)} \int_{0}^{x}(x-t)^{\alpha-1}(\tau-t)^{\alpha-1} d t
\end{aligned}
$$

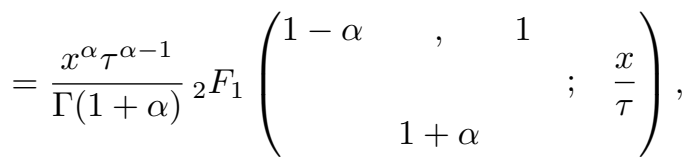

where ${ }_{2} F_{1}$ is the Gauss-hypergeometric function which can be defined by its Euler representation, see [12],

$$
{ }_{2} F_{1}\left(\begin{array}{ccccc}
a & , & b & \\
& & & ; & z
\end{array}\right)=\frac{\Gamma(c)}{\Gamma(b) \Gamma(c-b)} \int_{0}^{1} s^{b-1}(1-s)^{c-b-1}(1-z s)^{-a} d s .
$$

Thus, this problem has an infinite sequence of real negative eigenvalues. The Taylor expansion up to order 1 gives

$$
\left(T_{\alpha} y\right)(x)=\left.\left(T_{\alpha} y\right)(x)\right|_{\alpha=1}+\left.\left(T_{\alpha}^{(1)} y\right)(x)\right|_{\alpha=1}(\alpha-1)+R_{1}(\alpha)
$$


where the kernel $\left.\widetilde{G}_{\alpha}(x, \tau)\right|_{\alpha=1}=x \tau-\min (x, \tau)$ is the classical Green's function of the $\left(\mathcal{P}_{1}\right)$ problem corresponding to our data.

$\left.\widetilde{G}_{\alpha}^{(1)}(x, \tau)\right|_{\alpha=1}$ can be computed by integration by parts

$$
\begin{aligned}
& \left.\widetilde{G}_{\alpha}^{(1)}(x, \tau)\right|_{\alpha=1} \\
& =\left\{\begin{array}{c}
2 \tau+2(\gamma-1) x \tau-u(x)-u(\tau)+u(x-\tau) \\
+u(x \tau)-x u(1-\tau)-\tau(1-x) \\
2 x+2(\gamma-1) \tau x-u(\tau)-u(x)+u(\tau-x) \\
+u(\tau x)-\tau u(1-x)-x u(1-\tau)
\end{array}\right. \\
& \quad \text { if } 0 \leq \tau \leq x,
\end{aligned}
$$

where $u(x)=x \ln x$ and $\gamma$ is the Euler constant.

We remark that the linear operator associated to this Green's function which contains logarithmic functions is not connected to any classical differential operator. Finally, we have

$$
\lim _{\alpha \underset{<}{<}} \lambda_{1}^{-}(\alpha)=-\pi^{2} .
$$

Acknowledgments. We are grateful to the referees for their careful reading of the manuscript. Their comments, questions and some additional suggestions for references were of great importance in helping to improve the quality of the paper. We thank them warmly.

\section{REFERENCES}

1. O.P. Agrawal, Generalized variational problems and euler-lagrange equations, Comp. Math. Appl. 59 (2010), 1852-1864.

2. E. Bas, Fundamental spectral theory of fractional singular Sturm-Liouville operator, J. Funct. Spaces Appl. 2013 (2013), 1-7.

3. Sci. Tech. 37 (2015), 251-257.

4. E. Bas, R. Yilmazer and E.S. Panakhov, Fractional solutions of Bessel equation with $N$-method, The Scientific World J. Math. Anal. 2013, (2013), 1-8.

5. M.S. Birman and M.Z. Solomjak, Spectral theory of self-adjoint operators in Hilbert space, D. Reidel Publishing Company, Amsterdam, 1987.

6. N. Dunford and J.T. Schwartz, Linear operators, Vol. II, Spectral theory, self-adjoint operators in Hilbert space, Interscience Publishers, New York, 1963.

7. M. Klimek and O.P. Agrawal, Fractional Sturm-Liouville problem, Comp. Math. Appl. 66 (2013), 795-812. 
8. M. Klimek and O.P. Agrawal, On a regular fractional Sturm-Liouville problem with derivatives of order in $(0,1)$, Slovakia IEEE Explore Digital Library, (2012), $3284-289$.

9. M. Klimek and M. Blasik, Regular Sturm-Liouville problem with RiemannLiouville derivatives of order in $(1,2)$ : Discrete spectrum, solutions and applications, Lect. Notes Electr. Eng. 320 (2015), 25-36.

10. _ Regular fractional Sturm-Liouville problem with discrete spectrum: Solutions and applications, IEEE (2014), 1-6.

11. A.A. Kilbas, H.M. Srivastava and J.J. Trujillo, Theory and applications of fractional differential equations, Elsevier, Amsterdam, 2006.

12. N.N. Lebedev, Special functions and their applications, Prentice-Hall, Inc., New York, 1965.

13. M. Mckiernan, On the nth derivative of composite functions, Math. Notes 63 (1956), 331-333.

14. K.S. Miller and B. Ross, An introduction to the fractional calculus and fractional differential equations, Wiley and Sons, New York, 1993.

15. I. Podlubny, Fractional differential equations, Academic Press, San Diego, 1999.

16. M. Rivero, J.J. Trujillo and M.P. Velasco, A fractional approach to the Sturm-Liouville problem, Centr. Eur. J. Phys. 11 (2013), 1246-1254.

17. S.G. Samko, A.A. Kilbas and O.I. Marichev, Fractional integrals and derivatives, Theory and applications, Gordon and Breach Science Publishers, Amsterdam, 1993.

18. V.K. Tuan, Inverse problem for fractional diffusion equation, Fract. Calc. Appl. Anal. 14 (2011), 31-55.

19. J. Weidmann, Linear operators in Hilbert spaces, Springer-Verlag, New York, 1980.

Department of Mathematics, University of Tlemcen, B.P. 119-Tlemcen 13000, Algeria

Email address: fz_bensidhoum@yahoo.fr

Department of Mathematics, University of Tlemcen, B.P. 119-Tlemcen 13000, Algeria

Email address: h_dib@mail.univ-tlemcen.dz 